\title{
PostureCare - Towards a novel system for posture monitoring and guidance
}

\author{
Andreas Schrempf, Gerold Schossleitner, Thomas Minarik* \\ Michael Haller, Sabine Gross, Werner Kurschl ${ }^{* *}$ \\ * Upper Austria University of Applied Sciences, School of Applied Health and \\ Social Sciences, Medical Technology, Garnisonstrasse 21, A-4020 Linz, \\ Austria, e-mail: andreas.schrempf@fh-linz.at \\ ** Upper Austria University of Applied Sciences, School of Informatics, \\ Communications and Media, Softwarepark 11, A-4232 Hagenberg, Austria
}

\begin{abstract}
A regular office chair was equipped with force transducers, which allow to monitor and to guide the sitting behavior of a person during an office working day. Only four force transducers are required to estimate characteristic parameters for quantifying the biomechanical effect of a certain sitting posture. Based on these parameters, which rely on a simple biomechnical model, a posture cost function was proposed for classification of the sitting posture. Since for posture guidance the posture control system has to be known, a simple approach for identification of the controller parameters is proposed. A framework for designing effective exercises is presented and allows to assess different exercises for improving the person's sitting behavior. The proposed sensor concept of the chair as well the proposed biomechanical model are evaluated by means of laboratory experiments.
\end{abstract}

Keywords: Biomedical system modeling, Simulation

\section{INTRODUCTION}

The typical office workplace nowadays is a computer workstation where the average office worker spends about 50,000 hours seated in the course of his working life. As a consequence of this motion deficiency about $40 \%$ of the office workers develop back problems [Ertel et al., 1997]. Computer work typically results in low-level static loading of back, shoulder and neck muscles as well as in static spine loading. Prolonged, static sitting is associated with muscular disorders, the development of disc degeneration or the increase of spine stiffness [Kingma and van Dieën, 2009, Beach et al., 2005]. Dynamic sitting, thus a behavior where the person dynamically activates lumbar muscles and spine movement is increased in a physiologically reasonable range is beneficial and will help to prevent back pain.

For most people it is however, difficult to check their sitting behavior and posture while working concentrated on a task and people usually are not aware of the time they spend on work activities while sitting statically [Daian et al., 2007]. In order to improve the sitting behavior of office workers an intelligent office chair was developed, which allows classifying the sitting posture and the corresponding time the person sits statically in any position (Figure 1 depicts common sitting postures which appear during an office working day). The aim of the intelligent office chair is to guide the person through an effective feedback to a more dynamic and healthy sitting behavior. Once our intelligent chair, equipped with four force transducers, detects that the office worker is sitting "unhealthy", an alert is provided to direct a person away from this posture.

There are three possibilities to train users sitting ergonomically correct on chairs, including a) an ergonomic chair with unstable support, b) triggering a training session, and c) providing
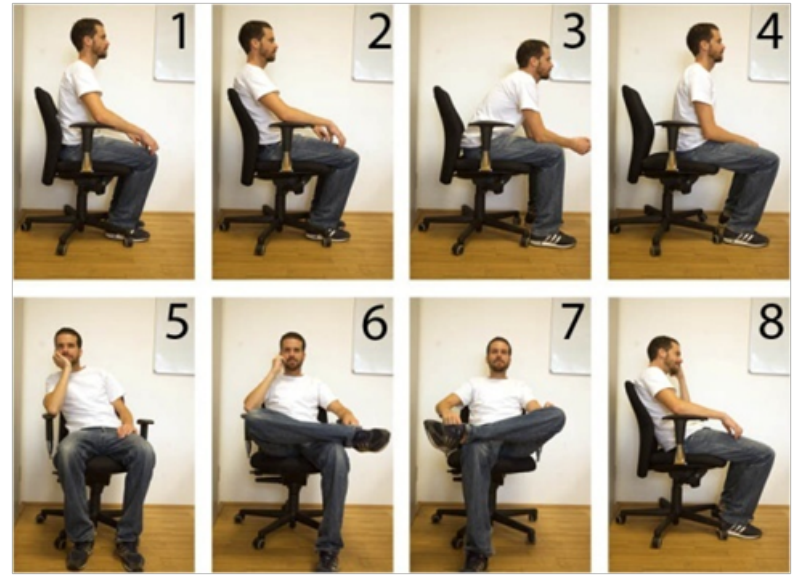

Figure 1. Sitting postures that usually appear during a working day. 1. upright, 2. leaning back, 3. leaning forward, 4. sitting at the front edge, 5. leaning right, 6. right leg crossed over left leg, 7. left leg crossed over right leg, 8. slouching.

direct guidance on the actual sitting position. First of all, an ergonomic chair with unstable support can be used, where users always have to balance their body on the chair which keeps them active (e.g. Haider - Bioswing). Another approach is to trigger users periodically to perform an exercise, where the users can use a regular office-chair. Zheng and Morrell [2010] propose an ergonomic chair which guides office workers to sit in a pre-defined position by providing a vibrotactile feedback. Force sensors placed on the sitting support as well as on the back rest of the chair are used to compute a feedback signal by using small vibration actuators. If the user is not sitting in a 
desired position, one or more actuators vibrate in order to direct the user away from this undesired position.

In contrast, our approach is not to exactly detect a certain posture but to estimate the biomechanical effect a certain sitting posture is responsible for. Hence we are going first to identify simple measurable parameters which are related to biomechanical factors related to the spine. Based on these parameters the approach is to classify the sitting posture with the help of a posture cost function. It turns out that one important parameter is the time a person sits statically. Another important parameter is the force acting on the spine which is closely related to the flexion and extension angle of the lumbar spine. Since e.g. a slouching position is more harmful to the spine than leaning back using the backrest (compare position 8 vs. position 2 in Figure 1) a cost function based on both, static sitting and flexion/extension angle is considered to be a proper approach for posture monitoring and classification.

\section{INTELLIGENT OFFICE CHAIR}

In contrast to Fenety et al. [2000] or Mutlu et al. [2007] our approach is to develop a simple, affordable approach which allows to use conventional office chairs. Our setup is based on a regular adjustable office chair, equipped with four independent, especially designed force transducers. The signal of the force transducers is digitized by a time-to-digital converter (ACAM Messtechnik $\mathrm{GmbH}$ ) and is then further processed and sent by a microcontroller (NEON, spantec $\mathrm{GmbH}$ ) via the wireless ANT $^{\mathrm{TM}}$ protocoll.

The four force transducers are located at each corner under the seating support, thus making it possible to compute the coordinates of the Center of Pressure $(C O P)$ with the reference frame located in the center of the seating support. The positive $x$-axis points towards the right side of a subject sitting on the chair and the $y$-axis faces towards the toes.

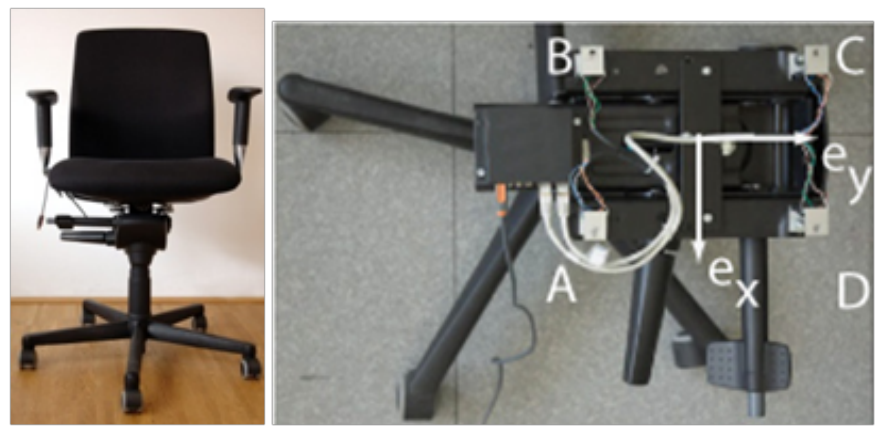

Figure 2. (left) The equipped office chair (right) Support frame of the chair. The 4 sensors are marked with the letters A$\mathrm{D}$, the reference frame is located in the center and labeled with ex and ey for the corresponding positive direction.

The position of the COP corresponding to the reference frame in Figure 2 can be determined by

$$
r_{C O P}=\left(\begin{array}{l}
C O P_{x} \\
C O P_{y}
\end{array}\right)=\frac{1}{F_{S}} \sum_{i=1}^{4} r_{i} F_{i}
$$

where $F_{i}$ denotes the force measured by the $i-$ th force transducer at position $r_{i}=\left(r_{i, x} r_{i, y}\right)^{T}$. The resulting force is given by $F_{S}=\sum_{i=1}^{4} F_{i}$. The coordinates of the COP vary according to the posture the person is sitting on the chair which in turn allows to classify the sitting posture and the time spent in the corresponding position.

The coordinates of the $C O P$ as well as the force $F_{S}$ acting on the sitting support are computed in real-time on the microcontroller and then they are sent wirelessly via the ANTprotocoll to any desktop computer or laptop equipped with an ANT USB-stick, which receives the data packets. A data server application based on a client/server architecture provides a realtime data stream via TCP/IP for further data processing with MatLab $^{\text {TM }}$. Since the data stream is provided via TCP/IP the data server and the client can be on different computers and locations. In MatLab ${ }^{\mathrm{TM}}$ a socket-connection to any (remote) TCP/IP data server based on Java provides the raw-data for further algorithm development as well as for measurement studies. A graphical user interface in combination with several underlying algorithms provides essential parameters for classification of the sitting posture as depicted in Figure 3.

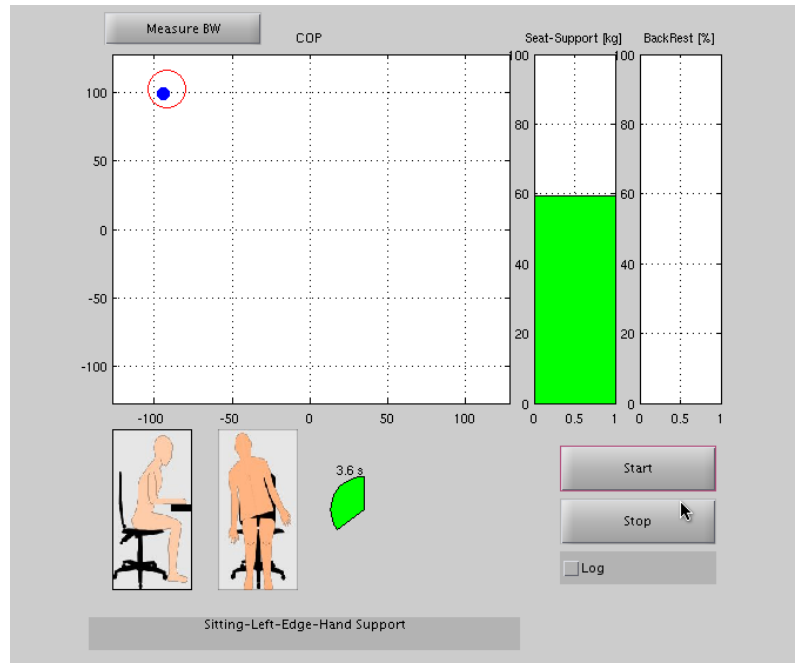

Figure 3. Matlab GUI: The coordinates of the $C O P$ are represented by a dot which moves within the area of the sitting support (upper left). Further parameters i.e. the force acting on the sitting support as well as the usage of the backrest are provided (bars on the right side).

\section{POSTURE MONITORING}

\subsection{Biomechanical Model}

In order the provide the relation between the sensor readings $C O P_{x}, C O P_{y}$ and $F_{S}$ of the intelleigent office chair and the human's posture, consider the simplified model of a sitting person as depicted in Figure 4. Hereby we assume, that the legs bended perpendularly. For small angles $\varphi_{x}(t)$ and $\varphi_{y}(t)$ the equations of motion of head, arm and trunk (HAT) are given by

$$
\begin{aligned}
& \Theta_{H A T} \ddot{\varphi}_{x}(t)=m_{H A T} g l_{C O M} \varphi_{x}(t)-M_{L x}(t)-M_{B}(t) \\
& \Theta_{H A T} \ddot{\varphi}_{y}(t)=m_{H A T} g l_{C O M} \varphi_{y}(t)-M_{L y}(t)
\end{aligned}
$$

where $\Theta_{H A T}$ denotes the corresponding inertia, $m_{H A T}$ the mass, $l_{C O M}$ the distance of the center of mass of HAT from the lumbar joint, $g$ the gravitation contant and $M_{L x}(t), M_{L y}(t)$ denote the components of the moment around the lumbar joint. The moment $M_{B}(t)=l_{B} F_{B}(t)$ denotes the support of the backrest. On the other hand the moment of the lumbar joint is in equillibrium with the moment generated by the gravity force $M_{G t}=$ 


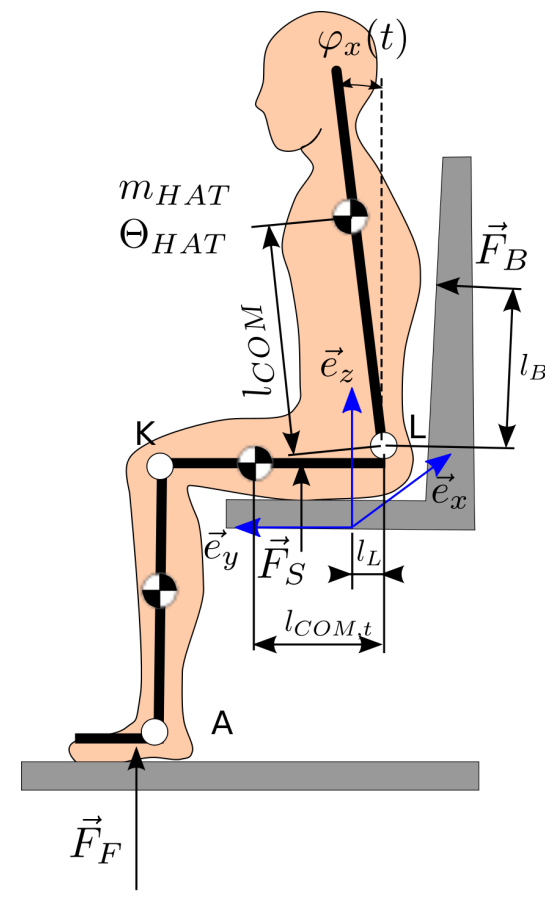

Figure 4. Simplified biomechanical model of a sitting person.

$-l_{C O M . t} F_{G t}$ of the thigh as well as by the moments generated by both, the sitting and foot support force such that

$$
\begin{aligned}
& M_{L x}(t)=\left(\operatorname{COP}_{y}(t)+l_{L}\right) F_{S}(t)+l_{t}\left(F_{F}(t)-F_{G s}\right)-l_{C O M, t} F_{G t}, \\
& M_{L y}(t)=\operatorname{COP}_{x}(t) F_{S}(t) .
\end{aligned}
$$

Hereby $l_{t}$ denotes the segement length of the thigh, $l_{L}$ the distance of the reference frame to the lumbar joint and $F_{G s}$ the gravity force of shank and foot. The gravity forces of shank and tigh can be expressed - by using anthropometric data - as a portion of the body weight i.e. $F_{G t}=a \cdot B W$ and $F_{G s}=b \cdot B W$. The location of the center of mass of the thigh is related to its segment length by $l_{C O M . t}=c \cdot l_{t}$. From the sensor readings of the four force transducers, the force acting on the sitting support $F_{S}(t)$ as well as the coordinates of the $C O P$ can be measured. If the body weight $B W$ of the person is known, the force acting on the foot support at any stationary point is given by $F_{F}=B W-F_{S}$. This in turn enables us to estimate the joint moment $M_{L}(t)$ by

$$
\begin{aligned}
& M_{L x}(t)=\left(C O P_{y}(t)+l_{L}-l_{t}\right) F_{S}(t)+\lambda l_{t} B W \\
& M_{L y}(t)=C O P_{x}(t) F_{S}(t)
\end{aligned}
$$

with $\lambda=1-b-c a$, where $\operatorname{COP}_{x}(t), C_{C O P_{y}}(t)$ and $F_{S}(t)$ are obtained by the sensor readings of the force transducers. The equations of motion of head, arm and trunk according to eq. (2) and eq. (3) form in case of small movements a linear system

$$
\frac{d x(t)}{d t}=A x(t)+B u(t)
$$

with the system matrices

$$
\begin{aligned}
A & =\left(\begin{array}{cccc}
0 & 1 & 0 & 0 \\
\frac{m_{H A T} g l_{C O M}}{\Theta_{H A T}} & 0 & 0 & 0 \\
0 & 0 & 0 & 1 \\
0 & 0 & \frac{m_{H A T} g l_{C O M}}{\Theta_{H A T}} & 0
\end{array}\right), \\
B & =\left(\begin{array}{cc}
0 & 0 \\
-\frac{1}{\Theta_{H A T}} & 0 \\
0 & 0 \\
0 & -\frac{1}{\Theta_{H A T}}
\end{array}\right),
\end{aligned}
$$

where the state is given by $x(t)=\left(\varphi_{x}(t), \omega_{x}(t), \varphi_{y}(t), \omega_{y}(t)\right)^{T}$ and the input vector is $u(t)=\left(M_{L x}(t)+M_{B}(t), M_{L y}(t)\right)^{T}$ with the joint moments provided by eq. (4) -(5). Changing the state of the body to any desired state $x_{r}=\left(\varphi_{x}, 0, \varphi_{y}, 0\right)$ is reflected by a change of the $C O P$-coordinates to

$$
\begin{aligned}
& C O P_{x}=k_{\varphi} \varphi_{y} \\
& C O P_{y}=k_{\varphi} \varphi_{x}+C O P_{y 0}-\beta(t) l_{B} .
\end{aligned}
$$

Hereby $F_{S}$ as well as $m_{H A T} g$ are expressed as a portion of bodyweight i.e. $F_{S} / B W=\sigma, m_{H A T} g / B W=d$ and hence the gain $k_{\varphi}$ is given by $k_{\varphi}=l_{C O M} d / \sigma$. The offset $C O P_{y 0}$ is given by $C O P_{y 0}=\left(1-\frac{\lambda}{\sigma}\right) l_{t}-l_{L}$. Further the support of the backrest $M_{B}(t)=l_{B} F_{B}(t)$ is expressed as a portion of the force $F_{S}(t)$ by $F_{B}(t)=\beta \cdot F_{S}(t)$ which leads to $M_{B}(t)=\beta l_{B} F_{S}(t)$.

Since in our situation the backrest is in fixed position the angle $\varphi_{x}$ is limited by $\varphi_{x, \text { min }}$. In case, where the backrest is not just used, the smallest possible shift is $C O P_{y}=k_{\varphi} \varphi_{x, \min }+C O P_{y 0}$. If the backrest is used the $C O P_{y}$ shifts towards more negative values. This in turn allows us to estimate the usage of the backrest only from $C O P_{y}$ data by

$$
\beta=\max \left(\frac{k_{\varphi} \varphi_{x, \text { min }}+C O P_{y 0}-C O P_{y}}{l_{B}}, 0\right)
$$

The angles $\varphi_{x}$ and $\varphi_{y}$ in turn can be estimated from eq.(7)-(8) as

$$
\begin{aligned}
\varphi_{x} & =\frac{C O P_{y}-C O P_{y 0}+\beta \cdot l_{B}}{k_{\varphi}}, \\
\varphi_{y} & =\frac{C O P_{x}}{k_{\varphi}} .
\end{aligned}
$$

The derived equations eq.(4)-(5) and eq.(9)-(11) form now the basis for the classification of different sitting postures.

\subsection{Posture Classification}

In contrast to Zheng and Morrell [2010] or Mutlu et al. [2007] our goal is not to exactly classify the sitting postures depicted in Figure 1, since a reliable classification requires additional sensors which for example are able to detect the curvature of the spine. Our goal was to develop an affordable sensor concept which in turn can be simply applied to any common office chair. We are using the following characteristic biomechanical parameters, which all can be estimated by means of the force measurements.

The lumbar joint moment $M_{L}$ provides a sensible biomechanical parameter which reflects the load acting on the spine. The higher the lumbar joint moment, the higher is the load acting on the spine segments and the vertebral discs. The load acting on the spine segments is further reflected by the flexion/extension 
angle $\varphi_{x}$ as well as $\varphi_{y}$. With increasing flexion angle $\varphi_{x}$ the tendons around the spine are stretched and as a consequence the load on the spine segments increases [Harrison et al., 1999]. Additionally the vertebral discs are more and more deformed anterior with increasing flexion angle, which is reflected by a higher load on the internal structure of the disc, especially on the annulus fibrosus which consists of several layers of collagen fibres in order to provide stability against bulging. On the other hand if the angle $\varphi_{x}$ becomes negative an extension of the spine occurs which can lead to high loads on the spine segments due to a direct contact between facet joints and the segment base. Hence the absolute values of both angles, $\varphi_{x}$ and $\varphi_{y}$ provide the first characteristisation parameters. Notice that these angles can be estimated from eq. (10)-(11).

Very important is the fact, that during a prolonged static sitting period the metabolism of the vertebral discs cannot work as required, since the transport of nutrients to the nucleus pulposus as well as the transport of waste out of the disc requires a periodic loading and releasing of the disc. Prolonged static sitting periods lead to a strongly reduced metabolism which in turns weakens the internal structure of the vertebral disc and makes it prone to failure [Adams and Dolan, 2005]. Hence the variance of the lumbar joint moments forms another parameter proposed for posture classification where the posture cost function used is

$$
J_{p}=\frac{1}{t_{N}-t_{1}} \sum_{i \in\{x, y\}}\left(C_{1}\left\|\varphi_{i}\right\|_{2}^{2}+C_{2} e^{-C_{3}\left\|\Delta \bar{M}_{L i}\right\|_{2}^{2}}\right)=J_{p \varphi}+J_{p M}
$$

with adjustable weights $C_{1}, C_{2}$ and $C_{3}$.

Hereby the vector

$$
\bar{M}_{L i}=\frac{1}{B W \cdot H}\left(\begin{array}{c}
M_{L x}\left(t_{1}\right) \\
M_{L x}\left(t_{2}\right) \\
\vdots \\
M_{L x}\left(t_{N}\right)
\end{array}\right)
$$

denotes the data vector of the normalized lumbar joint moment vector component $x$ in a moving window of length $N$. All other data vectors can be defined accordingly where $\Delta \bar{M}_{L i}$ is formed by $\bar{M}_{L i}-$ mean $\left(\bar{M}_{L i}\right)$ In order to provide an index which does not depend on anthropometric data, the joint moments are normalized by the product of body weight $B W$ and body size $H$. Notice, that the posture quality index depends only on the measurement data provided by the force transducers and hence

$$
J_{p}=J_{p}\left(C O P_{X}, C O P_{y}, F_{S}\right)
$$

according to equations (4), (5), (10) and (11) respectively. The higher $J_{p}$ the more "unhealthy" the sitting posture is considered to be.

\section{POSTURE GUIDANCE}

In order to remind users to do an exercise if a unhealthy posture appears, we implemented three different alert modalities (Digital Feedback, Physical Feedback, and Vibrotactile Feedback). An important fact ist that the optimal feeback should produce high awareness of the person sitting on the chair but with the requirement, that the feedback should not disturb the work flow too much i.e. the intrusion should be minimized. Using different feedback modalities, the aim is to implement a feedback, which allows to optimize awareness by minimizing intrusion. However, in the context of this paper or focus is to provide a framework for the implementation of exercises and/or games which allow to improve human's sitting behavior. Since any movement and hence change of the sitting posture incorporates the posture control system, first this control system is going to be modeled and analysed.

\subsection{Posture Control System}

Changing the position of the body requires to adjust the muscle forces around the lumbar joint to produce $M_{L x}$ and $M_{L y}$ correspondingly and further to stabilize the desired position. This control system is formed by the plant - the equations of motion of head, arm and trunk according to eq. (6). The actuators of the control system are lumbar flexors and extensors, which produce a moment around the lumbar joint. A first attempt is to model the controller of the posture control system by a linear state controller

$$
u(t)=-K x(t)+L r_{c}(t)
$$

where $u(t)=\left(M_{L x}(t), M_{L y}(t)\right)^{T}$ is the output of the controller, $K$ the corresponding gain matrix and $L$ the gain matrix for reference input $r_{c}(t)=\left(C O P_{x r}, C O P_{y r}\right)^{T}$. The controller is fed by the states of the plant $x(t)$ which represents the input from the vestibular, the proprioreceptive as well as from the visual system. In order to account for delays due to a limited speed of sensor integration in the central nervous system - the reaction time - there is a time-delay $T_{\text {delay }}$ between reference signal $r(t)$ and controller reference input $r_{c}(t)$

$$
r_{c}(t)=r\left(t-T_{\text {delay }}\right) .
$$

The controller parameters then can be estimated by solving a nonlinear optmization problem, where with an initial guess the controller parameters are obatined by iteratively performing a closed loop simulation in order to minimize the least squares error between simulated $C O P_{\text {sim }}$ and measured output $C O P$

$$
\hat{\Theta}=\arg \min _{\Theta}\left\|C O P_{\operatorname{sim}}(\Theta, t)-C O P(t)\right\|_{2}^{2}
$$

For achieving a minimal parametrization we propose that for each simulation iteration the controller gains are obtained by pole placement, where the desired poles $p_{x}=-a_{x} \pm b_{x} i$ and $p_{y}=-a_{y} \pm b_{y} i$ represent the optimization parameters $\Theta=$ $\left(a_{x}, b_{x}, a_{y}, b_{y}\right)^{T}$.

\subsection{Exercise Design Framework}

The posture monitoring system usually is designed to run in background and to monitor and to classify the user's sitting posture. However, once an unhealthy sitting posture is detected i.e. if the posture costfunction $J_{p}$ according to eq. (12) exceeds an predefined threshold $J_{p, \max }$ i.e.

$$
J_{p}\left(C O P_{x}(t), C O P_{y}(t), F_{S}(t)\right)>J_{p, \max }
$$

the aim is to provide an adequate feedback and to invite the sitting person to perform a well designed exercise and/or game, which allows to improve the person's sitting behavior - especially when used on a regular basis. In terms of control theory, the goal is to design an exercise which provides the user with a reference signal $r(t)=\left(C O P_{x r}(t), C O P_{y r}(t)\right)$ such that the posture costfunction $J_{p}$ is minimized. At the actual state of our research, a common framework for solving this problem is not availabe yet, but at least the proposed approach can be used to compare different exercises and provides a basis for improving exercise movements. 


\section{MEASUREMENT EXPERIMENTS}

In order to investigate the accuracy of the sensor concept and to evaluate the biomechanical model and the proposed framework for posture monitoring and guidance, different laboratory experiments were performed.

\subsection{Accuracy Analysis}

Since we used self customized force transducers the $C O P$ as well as the force $F_{S}$ acting on the sitting support were evaluated by measurements using both, the office chair and a Kistler force plate (Type 9281C, Kistler Instrumente AG Winterthur, Swiss). The office chair was positioned by using a connector plate on top of the force plate. A male $(B W=670 \mathrm{~N}, H=1.81 \mathrm{~m}$, age $=$ 29 years) was sitting on the office chair using a foot support not connected to the force plate. By using the MatLab GUI different reference positions were presented to the sitting person with the task to follow this reference. After synchronization of the measurement data, the computed $C O P$-values as well as the force $F_{S}$ were compared between both systems, where the force plate measurements are considered as reference.

It turns out that due to the simple construction the sensor readings are inaccurate and biased $(+9 \mathrm{~mm}$ in $x$-direction, $-43 \mathrm{~mm}$ in $y$-direction). After calibration the sensor readings are unbiased with a standard deviation of $\pm 7.4 \mathrm{~mm}$ in $x$-direction and $\pm 7.9 \mathrm{~mm}$ in $y$-direction. This corresponds to a relative $C O P_{x}$ error of $\pm 4.6 \%$ and a a relative $C O P_{y}$ error of $\pm 4.8 \%$ in relation to the measurement range of $165 \mathrm{~mm}$.

\subsection{Model Verification}

In order to verify the proposed biomechanical model a laboratory experiment performing controlled movements using the developed office chair was carried out. Hereby a person (male, $B W=670 \mathrm{~N}, H=1.81 \mathrm{~m}$ ) was sitting on the office chair with removed backrest. The chair was placed in front of the force plate which served as foot support. Further an inertial sensor system (XSens XM-B-XB3, Xsens Technologies B.V.) was used to measure the orientation of the torso. Figure 5 shows the comparison between the estimated flexion/extension angle $\varphi_{x}$ eq. (10) and the reference angle from the XSens system as well as the estimated force acting on the foot support $F_{F}(t)=$ $B W-F_{S}(t)$ and the reference measurement from the Kistler force plate. Additionally the estimated, normalized lumbar joint moments are depicted. Generally a good agreement between model and measurements can be observed. However, there are differences which are due the fact, that the used biomechanical model is a first simple approach. Since the curvature of the spine is flexible it is possible to remain at a predefined $C O P$ by a relative bending/rotation of lumbar spine segments, pelvis and thoracic spine. Further there are mesurement errors too, since the calibration was only performed for the hole chair. An individual calibration of each of the four force transducers will obviously improve the accuracy.

\subsection{Posture Classification}

With an experiment 6 participants ( 3 females, 3 males) with age $33.5 \pm 10.9$ years participated we tracked the participants' posture during a document-editing task. Thereby the participants had to extract words out of a text, where all spaces were removed. During the one-hour tracking session episodes
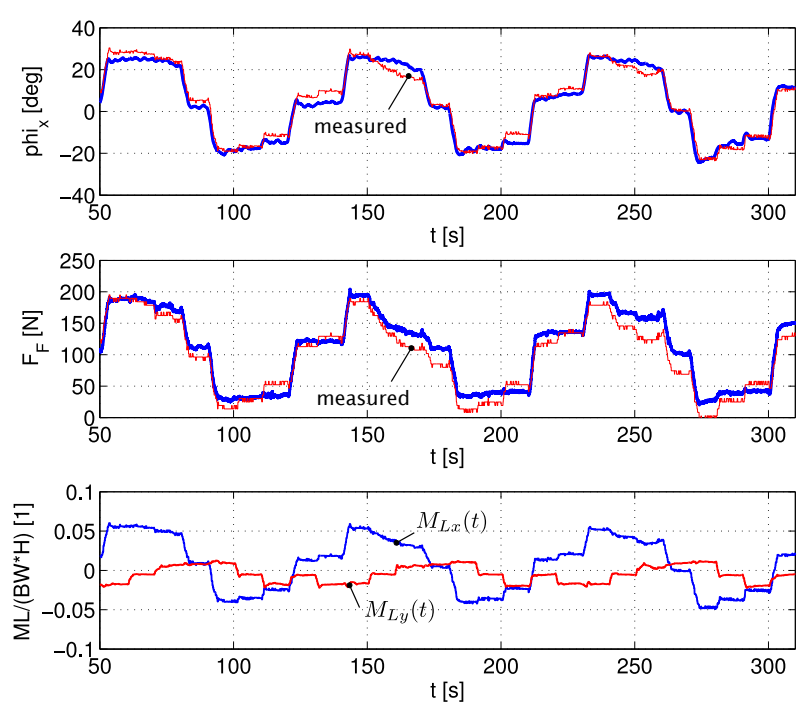

Figure 5. Comparison of estimated flexion/extension angle $\varphi_{x}(t)$, estimated foot support $F_{F}(t)$ and corresponding measurements as well as the estimated normalized lumbar joint moments $M_{L x}(t)$ and $M_{L y}(t)$.

of static sitting were identified, where the coordinates of the COP remain within a predefined region. During the one-hour session $5.8 \pm 3.9$ episodes of static sitting were identified lasting for $7.2 \pm 13.5$ minutes. Figure 6 compares the part $J_{p M}$ of the posture costfunction eq. (12) which is related to static sitting to the mean-time of static sitting episodes appeared for each participant during the study. For convenience the static

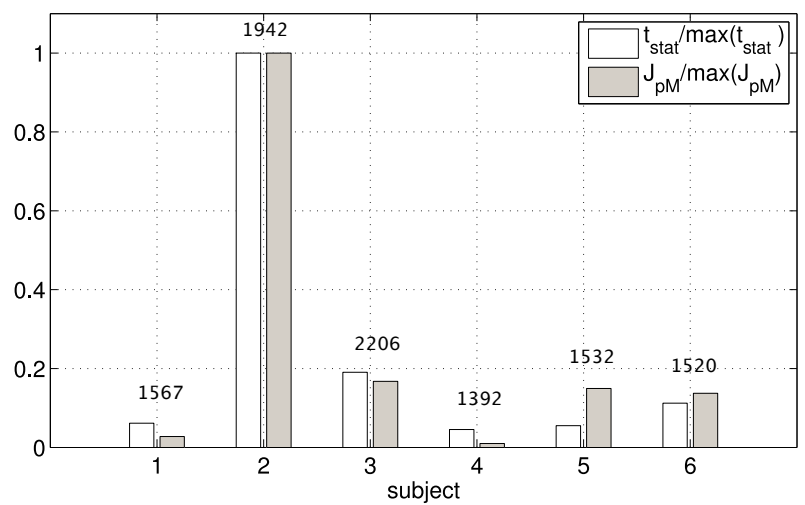

Figure 6. Comparison between static sitting time $t_{\text {stat }}$ and posture cost function $J_{p M}$ for an one-hour word editing task. Hereby the weights $C_{1}=1, C_{2}=150$ and $C_{3}=0.85$ were used. The number indicates how many words could be extracted by the participants.

sitting-time $t_{\text {stat }}$ as well as $J_{p M}$ is normalized by the maximum value appeared during the study. It can be concluded, that there is a good agreement and the posture cost function is able to quantify static sitting. Participants who managed to extract a higher number of words were sitting more statically than others.

\subsection{Posture Control System}

In order to obtain the controller gains of the posture control system, a laboratory experiment with controlled movements was carried out. Thereby the subject has to control the coordinates of the $C O P$ according to a given reference. The desired position was presented to the subject via a simple MatLab ${ }^{\mathrm{TM}}$ 
GUI where the subject has to reach as fast as possible the desired position. During this experiment all relevant variables were measured with the chair. The time-delay $T_{\text {delay }}$ was estimated from measurement data by building the mean response out of several trials and by identifying the time-delay (Figure 7). For control of the anterior-posterior movement of the body
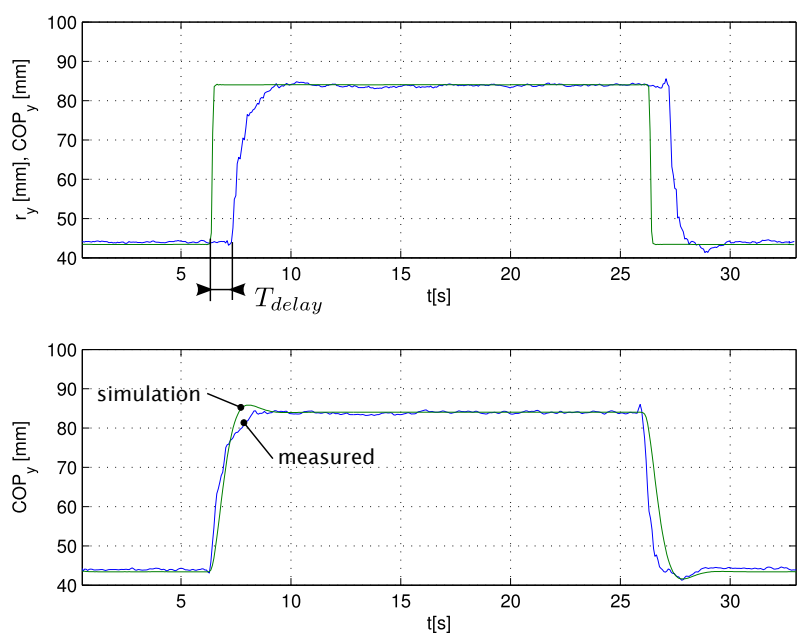

Figure 7. Estimation of the reaction-time and the controller parameters by means of an iterative optimization routine. Both plots show the mean sequence out of 10 times repeated controlled movements.

the reaction time was $T_{\text {delay }}=0.85 \mathrm{sec}$. where the closed loop poles after optimization were $p_{x}=-1.75 \pm 1.79 i$. For mediallateral movement the closed loop poles are $p_{y}=-1.97 \pm 1.74 i$, where the reaction time was identical.

\subsection{Posture Guidance}

Once a certain threshold for the posture cost function $J_{p}$ will be exceeded a feedback will remind the person to change the postition or to perform an exercise; hence the system should guide the person to healthier sitting. However, how to design an exercise or a game? Observing the posture cost function components $J_{p M}$ - which corresponds to "static" or "dynamic" sitting - and $J_{p \varphi}$ - which reflects the load on the spine - in Figure (8), the aim is to design exercises and games which allow the minimize the cost function $J_{p}$. One possible exercise

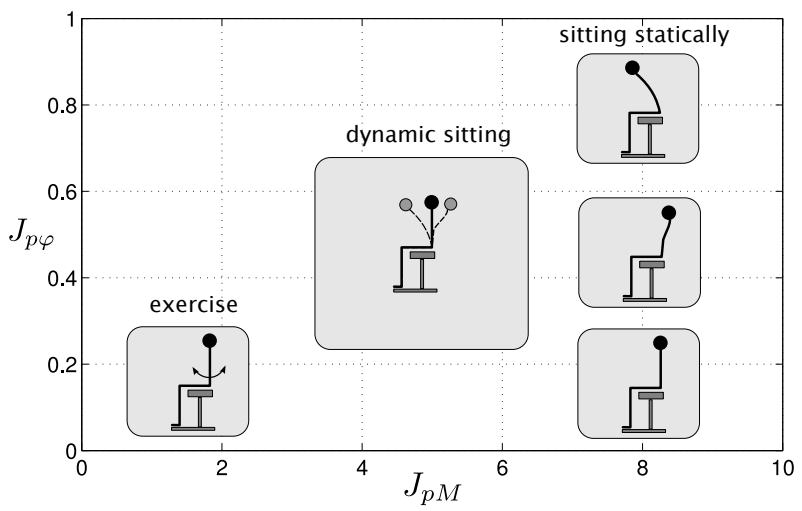

Figure 8. The components of the posture cost function $J_{p}$ for static sitting in different positions, for dynamic sitting and for performing the exercise "pelvic rocking".

is "pelvic rocking" where the pelvis is tilted periodically - in a

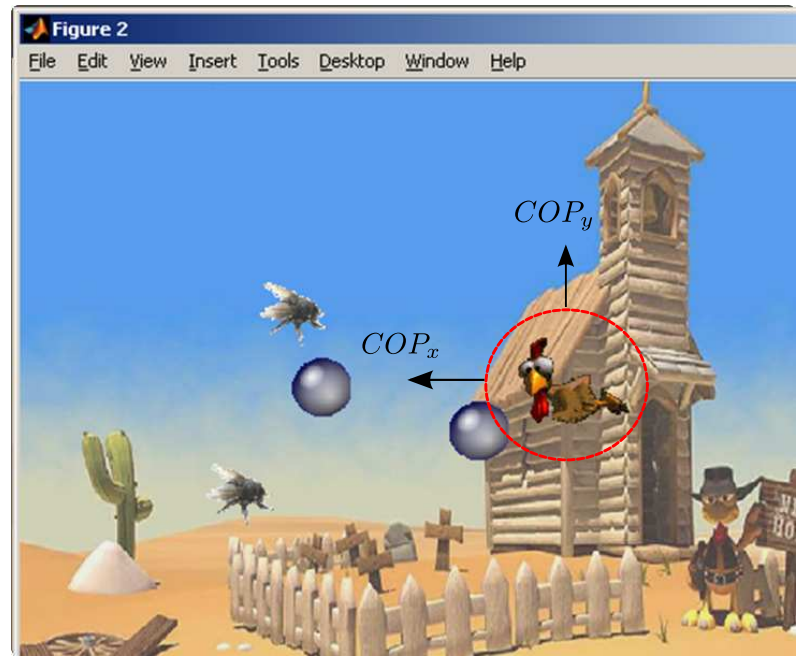

Figure 9. Simple game for learning the exercise "pelvis rocking" by playing. The chicken has to be controlled by rocking the pelvis in order to catch the flies and not to be hit by a cannonball.

small range of motion - by contracting deep abdominal muscles while the hands are resting on the knees. The same exercise can be also performed by playing a game. Figure 9 shows a simple game, where a chicken (which is controlled by the COP of the chair) has to avoid to be hit by a cannonball. The controll of the COP has to be performed by a "plevic rocking" like movement in both directions, vertically and horizontally respectively.

\section{CONCLUSIONS}

It turns out that the sensor concept of the developed intelligent office chair equipped with only four force transducers in combination with a simple biomechanical model is able to estimate and/or to measure the lumbar joint moment, the flexion/extension angle, the foot-support, the back rest usage as well as the force acting on the sitting support. With these parameters the classification of the sitting posture can be performed using the proposed posture cost function, which especially accounts for static sitting periods. The propsed framework is a first but reasonable approach for a systematic development of posture guidance algorithms in order to improve the sitting behavior of office workers in a long term-view and may help to prevent office workers from low back pain, which is a major problem in industrialized countries.

However, the sensor concept as well as the biomechanical model can be improved by e.g. an individual calibration of the force transducers or a bimechanical model using more segements which allow to consider a more complex shaping of the spine. Further research in this context will mainly concern the improvement of the whole framework and the intensive evaluation of the office chair in a long-term study.

\section{ACKNOWLEDGEMENTS}

The authors greatly acknowledge the financial support of the government of Upper Austria and the Upper Austria University of Applied Sciences. This publication is part of the project "PostureCare". Special thanks to Armin Blaha and Stefan Leipold, both spantec GmbH, for supporting us with the NEON device and ANT. 


\section{REFERENCES}

Michael A. Adams and Patricia Dolan. Spine biomechanics. Journal of Biomechanics, 38(10): 1972 - 1983, 2005. ISSN 0021-9290. doi: DOI: 10.1016/j.jbiomech.2005.03.028.

Tyson A C Beach, Robert J Parkinson, J. Peter Stothart, and Jack P Callaghan. Effects of prolonged sitting on the passive flexion stiffness of the in vivo lumbar spine. Spine J, 5(2): 145-154, 2005. doi: 10.1016/j.spinee.2004.07.036. URL http://dx.doi.org/10.1016/j.spinee. 2004.07.036.

I. Daian, A. M. van Ruiten, A. Visser, and S. Zubic. Sensitive chair: a force sensing chair with multimodal real-time feedback via agent. Proceedings of the 14th European conference on Cognitive ergonomics, pages 163-166, 2007. doi: http://doi.acm.org/10.1145/1362550.1362583.

M. Ertel, G. Junghanns, E. Pech, and P. Ullsperger. Effects of vdu-assisted work on health and well-being. Research Report 762, Federal Institute for Occupational Safety and Health (BAuA), 1997.

P. A. Fenety, C. Putnam, and J. M. Walker. In-chair movement: validity, reliability and implications for measuring sitting discomfort. Appl Ergon, 31(4):383-393, Aug 2000.

Donald D. Harrison, Sanghak O. Harrison, Arthur C. Croft, Deed E. Harrison, and Stephan J. Troyanovich. Sitting biomechanics part i: Review of the literature. Journal of Manipulative and Physiological Therapeutics, 22(9):594 609, 1999. ISSN 0161-4754. doi: DOI: 10.1016/S01614754(99)70020-5.

Idsart Kingma and Jaap $H$ van Dieën. Static and dynamic postural loadings during computer work in females: Sitting on an office chair versus sitting on an exercise ball. Appl Ergon, 40(2):199-205, Mar 2009. doi: 10.1016/j.apergo.2008.04.004. URL http://dx.doi.org/10.1016/j.apergo.2008.04.004.

Bilge Mutlu, Andreas Krause, Jodi Forlizzi, Carlos Guestrin, and Jessica Hodgins. Robust, low-cost, non-intrusive sensing and recognition of seated postures. Proceedings of the 20th annual ACM symposium on User interface software and technology, pages 149-158, 2007. doi: http://doi.acm.org/10.1145/1294211.1294237.

Ying Zheng and J.B. Morrell. A vibrotactile feedback approach to posture guidance. Haptics Symposium, 2010 IEEE, pages 351 -358, mar. 2010. doi: 10.1109/HAPTIC.2010.5444633. 